\title{
Design and Implementation of Yak Growth Information System Based on B/S Architecture
}

\author{
Junqiang $\mathrm{Li}^{1,}$, , Xiaowei Bai ${ }^{1}$, Cai Wen ${ }^{1}$ and Wei Xiang ${ }^{1, b^{*}}$ \\ ${ }^{1}$ College of Electrical and information Engineering, Southwest University for Nationalities of China, \\ Chengdu, Sichuan, 610225, P.R. China \\ a676376198@qq.com, b3730544@qq.com \\ ${ }^{*}$ The corresponding Author
}

Keywords: PHP; HTML; MySQL; B/S architecture; The yak growth information system

\begin{abstract}
PHP is an open source general-purpose computer scripting language and especially suitable for network development, and can be embedded in the use of HTML[1]. In this paper, the author used PHP script language and MySQL database to design and implement the yak growth information system based on $\mathrm{B} / \mathrm{S}$ architecture. The system is easy for the yak researchers and the yak breeding personnel to operate and manage.
\end{abstract}

\section{Introduction}

In recent years, yak market in China is developing rapidly, product output continued to expand, the national industrial policy is to encourage yak industry to develop high-tech product, domestic enterprises is increasing investment projects. Investors are more and more concerned about the yak market, which makes it concerned by the parties gradually. However, due to poor marketing convergence in the yak sales area, which restricts its long-term development. What's worse, the basic condition in the origin of the yak is poor. The large investment, the inconvenient traffic, the complex operation and other issues puzzle a lot of farmers though they are very interested in the improvement of yak. Similarly, yak breeding personnel or researchers are often lack of yak growth information, resulting in slow yak growth in yak breeding area.

In view of the above problems, this paper put forward to using the web page technology to design and implementation the $\mathrm{B} / \mathrm{S}$ structure of yak growth information system. Using the system to manage the growth information of yak could greatly reduce the time and effort for the relevant personnel. Moreover, because of the good stability of the system, it can guarantee the security and reliability of the relevant information.

\section{B/S Structure}

$\mathrm{B} / \mathrm{S}$ structure stands for the browser and server structure[2-3], as shown in fig. 1. It is an improved structure to $\mathrm{C} / \mathrm{S}$ structure with the rise of the Internet technology. What's more, it is a new software system construction technology combined with a variety of Script languages (such as VBScript, javascrip) and ActiveX technology, which mainly used the mature WWW browser technology[4].

It has 3-tier construction, which are: presentation layer, processing layer and data layer. Presentation layer is a browser. Its main function is to show the user interface but not to deal with the business. The main function of the processing layer is to receive the task request of the client browser, and to execute the corresponding transaction processing program according to the corresponding request type. The main function of the data layer is to receive the data manipulation request from the server to the database server. The database server complete data query, delete, modify and some other operations, and then submit the results of the data processing to the server. [5]

$\mathrm{B} / \mathrm{S}$ structure is a kind of network structure mode after the rise of WEB. The WEB browser is the main application software of the client. As long as we install a browser such as Internet Explorer or 
Mozilla Firefox on the client in B/S structure, customers do not need to install the user interface applications. Therefore, it simplifies the operation of the client. B/S structure is based on the TCP/IP which is an open network standard protocol, realizing completely the access to the computer and all kinds of resources on the network cross platform. So it has a strong system independence and platform independence. This would greatly simplify the client computer load, alleviate the cost and workload of system maintenance and upgrade and reduce the total cost of ownership (TCO).

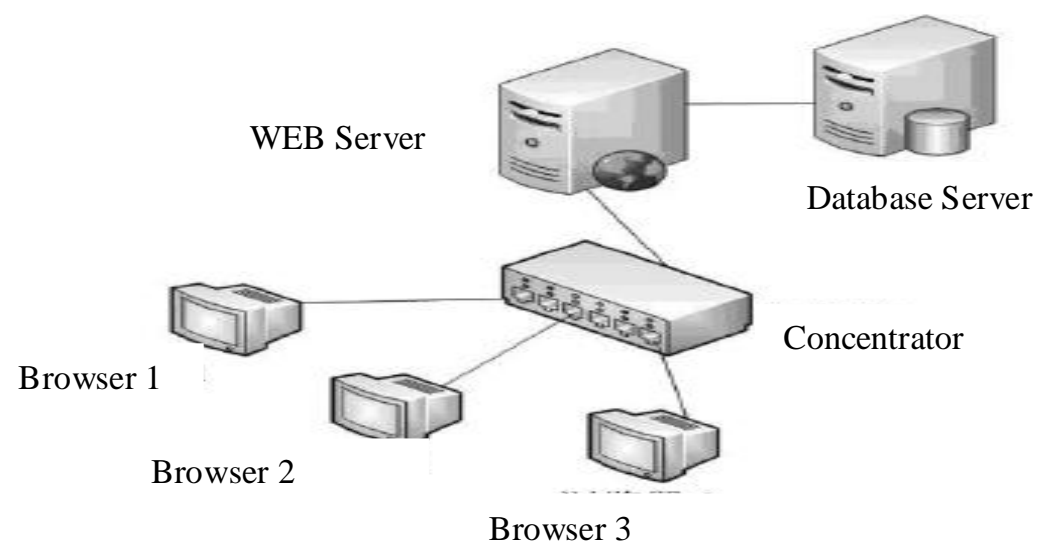

Figure 1. The B/S structure

\section{MVC Design Pattern}

The whole system uses the MVC(Model-View-Controller) design mode. The design idea is to separate the input, processing and output flow of an application in accordance with the model, view and controller way. [6] In this way, an application is divided into three core components: model (M), view $(\mathrm{V})$ and controller $(\mathrm{C})$. Each of them processes its own tasks. The flow chart is shown in fig. 2.

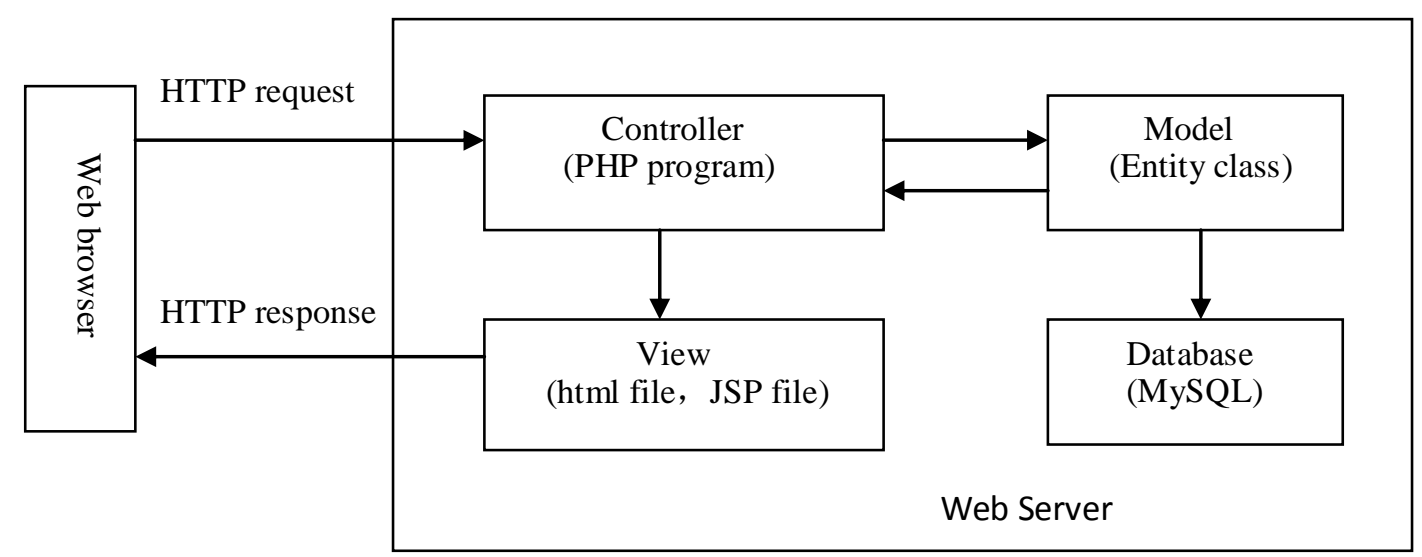

Figure 2. The flow chart of MVC design pattern

View: View is the interface that the user sees and interacts with. For the Web application of this system, view is the interface composed of HTML element. Actually, there is no real processing occurs in the view. It can only display the static page and collect the input data.

Model: Model represents for the business data and the business rules, which can interact with the background database. Model has the most processing tasks in the three part of MVC. MVC does not provide a design method for the model, but only tells the user that they should organize and manage these models[7]. Model accepts data from view requests and returns back the results of the final processing. The data returned by model is neutral and independent of the data format, so a model can provide data for multiple views. Because the code for the model only needs to be written only once and then can be reused by multiple views, model can reduce the duplication of code. 
Controller: Controller accepts the user's input, and then matches model and view to complete the user's needs together. Specifically, when the user clicks on the hyperlink in the Web pages and sends the HTML form data, controller will firstly receive the request and choose acceptable model to respond to user requests, and then determine the appropriate view to display the data from model[8]. Controller itself does not output anything and not do any data processing in the process.

The system is divided into MVC 3-tier construction[9], which has a strong portability and expansibility. Therefore, the way not only can make the user easy to debug and modify, but also make the whole system easy to maintain.

\section{Requirement Analysis}

The system uses the object-oriented analysis method to divide the function of the yak growth information system. Thus, we can divide the needs into the requirements for yak breeding staff, general manager and super administrator. Specific authorities the author divided are as follows:

(1)Yak breeding staff

(1) Modify their own personal information.

(2)Input the daily growth information of their own breeding yak to the database, and then add, delete, change and check it.

(2)General manager

(1)Achieve all functions of yak breeding staff.

(2) View the growth information of all yak breeding staff, and then increase, delete and modify it.

(3)Manage all yak breeding staff's information.

(3)Super administrator

(1)Achieve all functions of the general manager.

(2)Manage the general manager's information.

(3)Distribute relevant permissions to each user, so that every user can achieve different functions in accordance with their respective requirements.

\section{Database Design}

Database design refers that we construct the best database model with regard to a given application environment, establishing database and its application system so that it can effectively store data and meet the application requirements from a variety of users. [10] The database for yak growth information system consists of 7 tables: table of yak population, table of yak head, table of county, table of township, table of yak basic information, table of yak growth information, table of yak relationship.

(1)Table of yak population: Population ID, population name.

(2)Table of yak head: Yak head ID, the yak head's name, town ID that yak head belonging to.

(3)Table of county: County ID, the name of county.

(4)Table of township: Township ID, the name of the township, county id that township belongs to.

(5)Table of yak basic information: Yak ID, yak head ID, sex, population ID, birthday.

(6)Table of yak growth information: Node ID, yak ID, body height, body length, body weight, heart girth, cannon circumference, test date, image path.

(7)Table of yak relationship: Node ID, yak ID, mother ID, children ID

\section{System Design}

Technical Analysis of the System. The author used the HTML, CSS and JavaScript three languages to develop the front-end system, and used the PHP scripting language and the MySQL database to develop the back-end system. It is worth mentioning that, when the traditional client sends a request to the background server by way of a form or a button on the page, although it may 
be just little information transfer, it will lead to the client browser reload the entire page and refresh the full screen. Users will wait for the refreshed page frequently in the operation process. That causes a lot of trouble to the user ${ }^{[11]}$. Therefore, the author used the Ajax technology. This technology is combined with Java, XML and JavaScript programming technology, which can make the Web pages repeat loading without frequently interrupted, achieve dynamic interactive updates and significantly improve the user's experience. Its working principle is shown in Fig. 3:

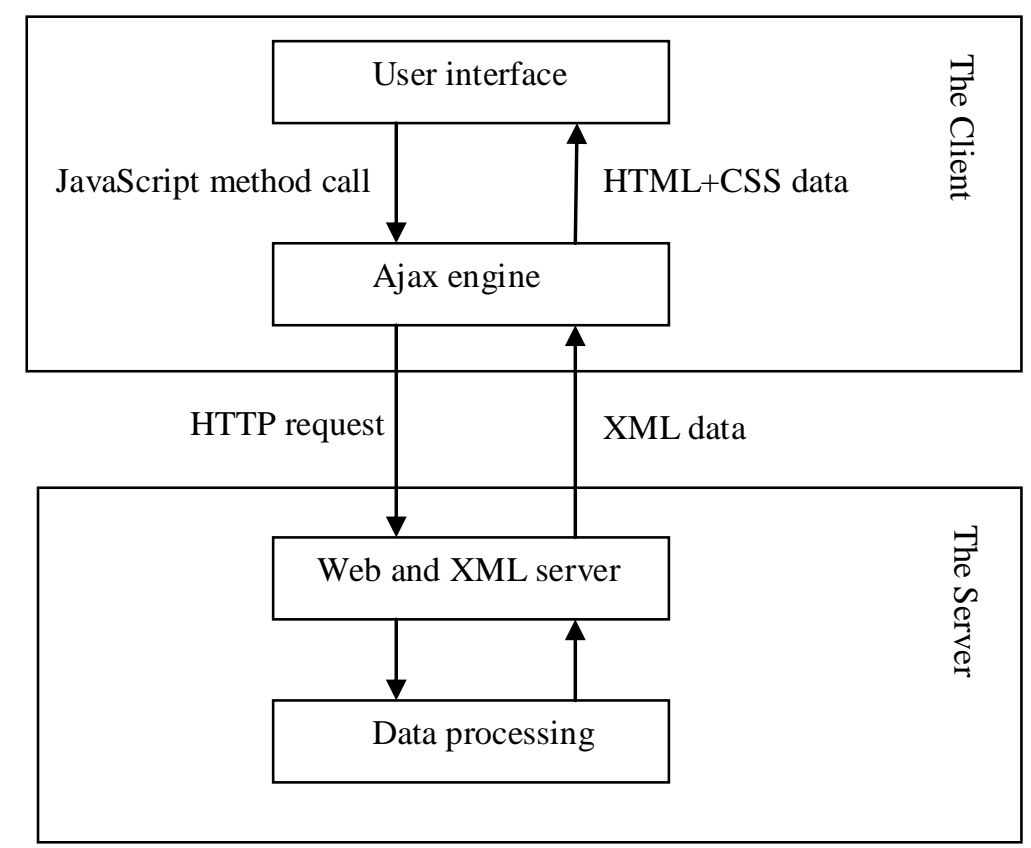

Figure 3. Ajax working principle

\section{Function Description Of the System}

The whole system is divided into the foreground and the background, which is shown in Fig. 4. When the input account password corresponds to the foreground, the system will jump to the foreground page. When the input account password corresponds to the background, the system will jump to the background page.

When the front-end users enter into the front page, they can start to manage their own personal information and their own yak information. Users can modify their own password and personal information. What's more, users can add, delete, change and check yak information to meet all basic requirements what users need to do for yak information management. The flow chart of the front-end operation is shown in Fig. 5.

The back-end users are divided into general manager and super administrator. When the input password corresponds to general manager, the background pages only show three parts: personal information, yak information and the yak breeding staff's information. When the input password corresponds to the super administrator, apart from the three parts for general manager interface that the background pages will display, there will be added access control and information of general managers two modules. The flow chart of the back-end operation is shown in Fig. 6. 


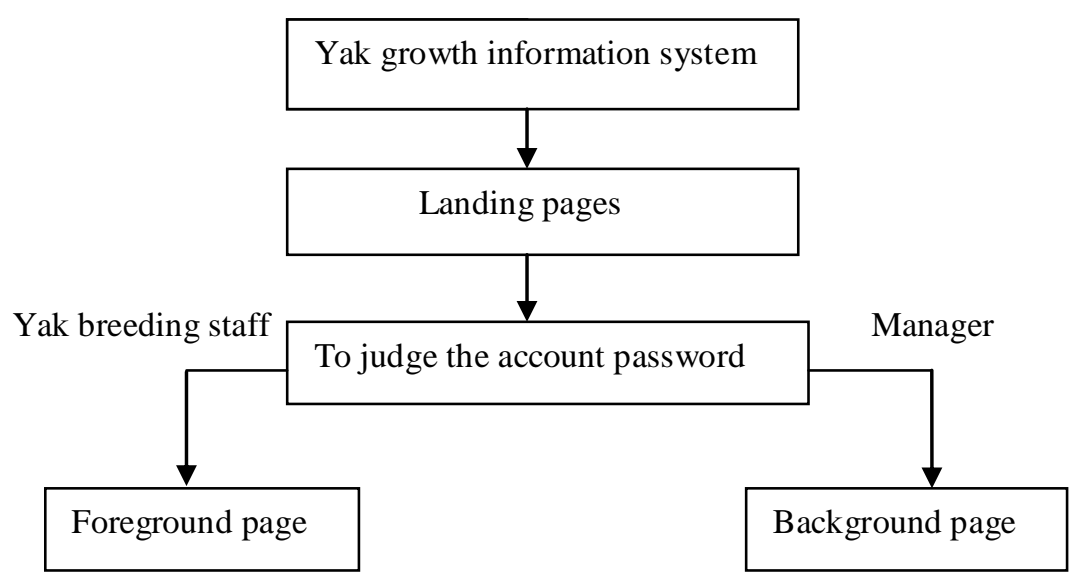

Figure 4. The flow chart of yak management system design flow

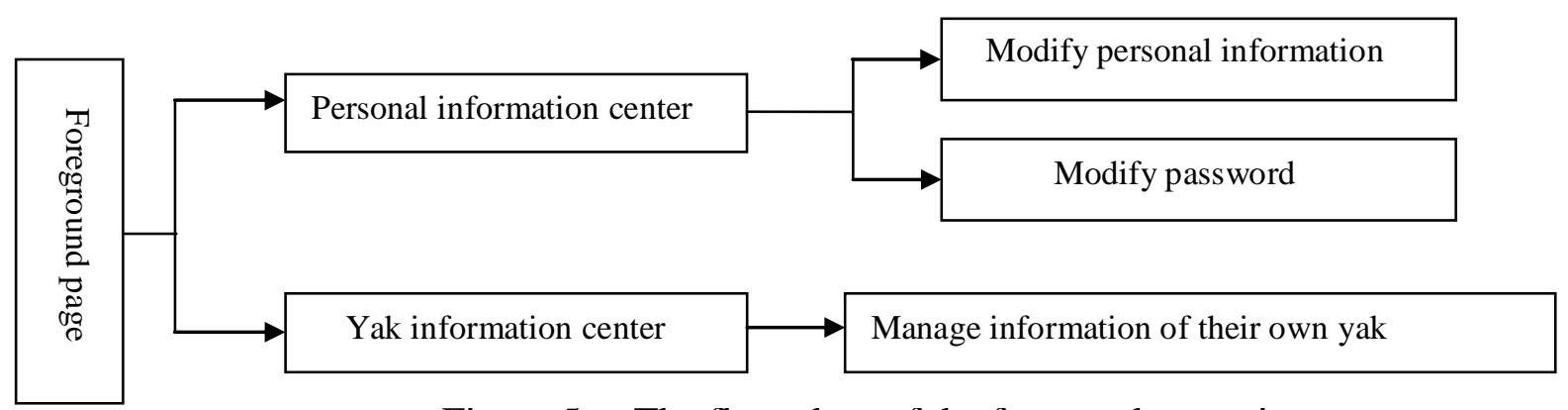

Figure 5. The flow chart of the front-end operation

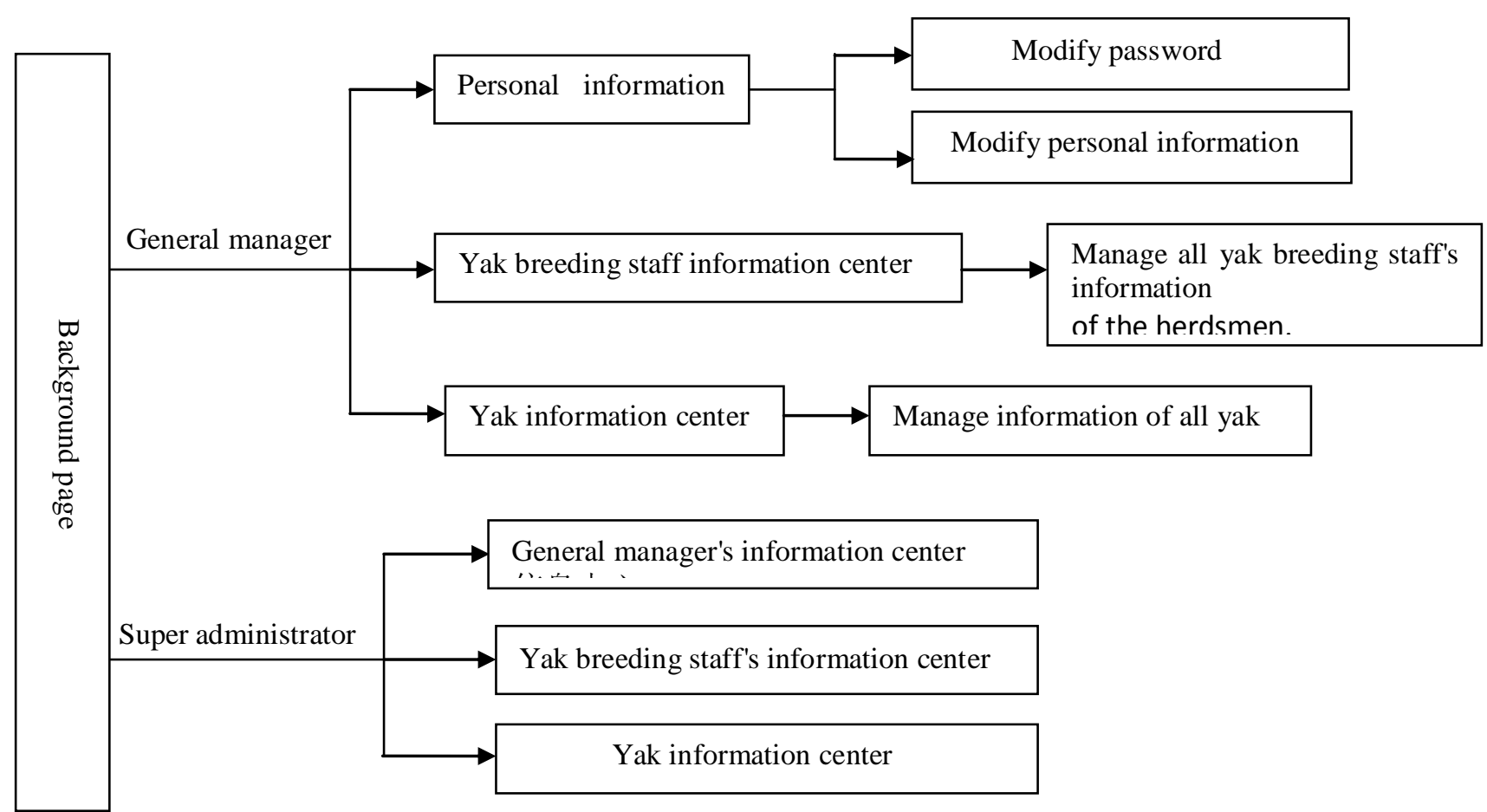

Figure 6. The flow chart of the back-end operation 


\section{Summary}

This paper designs a yak growth information system, gives the overall structure of the system and describes in detail the function of each module. The system is based on the B/S architecture. The front end uses a variety of languages such as HTML, CSS and JavaScript to develop, and the background uses the PHP language and MySQL database to develop. The system interacts with the users by the way of Web. The whole system is mainly suitable for yak breeding staff, yak researchers and other related personnel. It can greatly facilitate the related personnel to manage yak information, improve the development efficiency and expand and maintain the system. The system is stable during the testing process, which reduces the relevant personnel's workload and improves the accuracy of the data.

\section{Acknowledgements}

This work was financially supported by Innovative Research Team of the department of Sichuan Province(15TD0050) and SWUN students' innovative training program (No.S201610656066).

\section{Reference}

[1] Ji Qi. PHP Website Development Technology for Small and Medium Enterprises [D]. Jilin University, 2012.

[2] ZHAO Hong-wei, QIN Chang-ming. Research on Software Design Based on B / S 3-Tier Structure [J]. RESEARCH AND EXPLORATION IN LABORATORY, 2011, (07):64-66.

[3] HOU Shu-ying. Comparison to Advantage of B/S Pattern and C/S Pattern [J]. Journal of Shenyang College of Education, 2007,(02):98-100.

[4] CAI Chang-an, WANG Ying-ying. Comparison and Choice of B/S and C/S Mode [J]. Journal of Weinan Teachers College, 2006,02:47-50+74.

[5] Li Yunyun. Discussion on the Architecture of B/S and C/S[J]. Friends of Science Amateurs, 2011, 01:6-8.

[6] WANG Junfang, LI Yinfeng, WANG Chi. Research on ThinkPHP Framework Based on the Mode of MVC [J]. Electronic Sci.\&Tech,2014,04:151-153+158.

[7] Li Xia. the Theory and Realization of MVC Design Pattern[D].Jilin University,2004.

[8] DAI Yi-ping. Application of MVC Design Pattern in PHP Development [J]. Computer and Modernization, 2011, (03):33-37+43.

[9] MA Li,FENG Lei. Based on the MVC triple-layer structure art design and implementation of repository [J]. Electronic Design Engineering, 2016, (15):85-87.

[10] Wang Liang. The Design and Implementation of RSS Read System Based on ThinkPHP[D]. Huazhong University of Science and Technology, 2012.

[11]XIONG Wen, XIONG Shu-hua, SUN Xu, ZHANG Zhao-yang. Application Research of Ajax in web 2.0 Website Design [J].COMPUTERTECHNOLOGY AND DEVELOPMEN, 2012,03: $145-148$. 